\title{
Development of natural bitumen (bituminous sands) deposits based on the technology of hydraulic mining by boreholes
}

\author{
Larysa Pedchenko ${ }^{1}$, Nazar Pedchenko ${ }^{1}$, Andrii Manhura ${ }^{1}$, and Mykhailo Pedchenko ${ }^{1 *}$ \\ ${ }^{1}$ Poltava National Technical Yuri Kondratyuk University, Department of Oil and Gas Exploitation \\ and Geotechnics, 24 Pershotravnevyi Ave., 36011 Poltava, Ukraine
}

\begin{abstract}
The modern state and trends in the development of technologies for mining the heavy oil and native bitumen deposits have been analyzed. It has been set that presently known technologies for heavy oil and natural bitumen extraction do not cover the entire depths range of their occurrence. The main possibility of the development of heavy oil and natural bitumen deposits on the basis of technology by hydraulic mining by boreholes has been substantiated. The peculiarities of this technology are analyzed. The ways of its main elements adaptation for mining the natural bitumen from bituminous sands deposits are offered. The principal scheme has been substantiated for a method of mining the natural bitumen on the basis of hydraulic mining by boreholes. The thermodynamic processes occurring in the productive formation during its drilling with the simultaneous circulation of the heat-transfer medium have been analyzed. A mathematical model has been developed of the thermal penetration process into the productive formation for the time interval from the beginning the borehole drilling to the beginning the rock disintegration. Also, it has been solved the task of stationary mode in the borehole at the stage of its drilling.
\end{abstract}

\section{Introduction}

Easily available oil and natural gas reserves are constantly being exhausted. At the same time, the demand for gas and oil products is growing rapidly. The development of technologies and the growth of energy prices allow companies to make profitable extraction of non-traditional hydrocarbons. According to preliminary estimates, the resources of nontraditional sources of energy raw materials much exceed the resources of traditional hydrocarbons deposits. One of the strategic reserves of hydrocarbons are heavy oil and natural bitumen $[1,2]$.

Oil sand, also known as bituminous sand or tar sand, is composed of bitumen (heavy viscous oil), water, sand and clays. The formation of bitumen is believed to relate to the aerobic biodegradation and gradual transformation of lighter crude oil [3]. Highly viscous oils and natural bitumens are colloidal systems which have an amorphous structure. A

\footnotetext{
* Corresponding author: pedchenkomm@ukr.net
} 
typical composition of an oil sand ore is about $10 \mathrm{wt} \%$ of bitumen, $85 \mathrm{wt} \%$ of solids (sands and clays) and $5 \mathrm{wt} \%$ of water.

Takamura (1982) proposed a model for the microscopic structure of Athabasca oil sands [4]. The three components are all intermixed, with a thin $10 \mathrm{~nm}$ water film between the bitumen and sand particles, see Fig. 1.

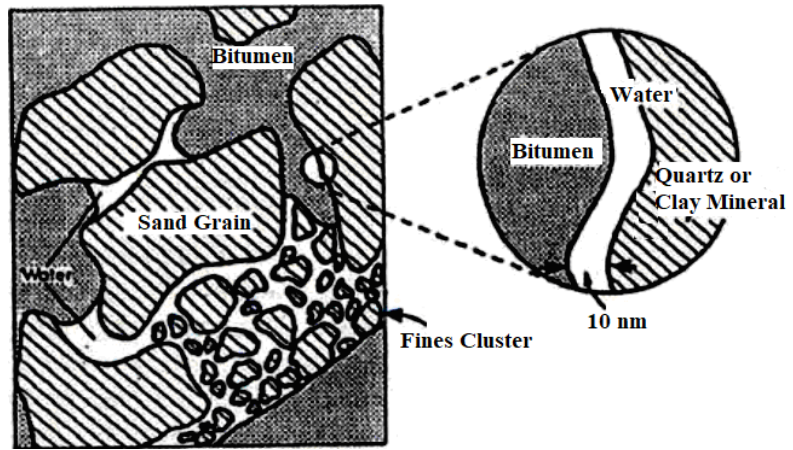

Fig. 1. The classical model of the microscopic structure of Athabasca oil sands proposed by [4].

About 600 deposits of oil sands in 23 countries are known. The largest world deposits are in Canada, Venezuela, Madagascar, USA and Russia [5]. According to the ERCB report (2011), the Canadian deposits of oil sands contain an estimated 1.7 trillion barrels (initial volume in-situ) of crude bitumen. About 1.3 million barrels of oil are produced every day from Athabasca oil sands.

Today, the development of bituminous sands deposits is carried out in the fields of Canada. However, the methods and approaches used in the extraction of traditional oil cannot be directly implemented on high-viscosity oil and natural bitumen deposits. For successful recovery, bitumen is to be purified from the sand grains and water matrix. The previous researchers have shown that a multitude of parameters are equally important for the bitumen recovery $[6,7]$.

This process requires a significant amount of energy consumption. Nevertheless, taking into account the present oil prices, this production is profitable.

The current industrial practice for bitumen recovery is based on two methods: surface and in-situ mining. Given the physical and chemical properties of natural bitumen, the application of methods that maximally reduce its viscosity, is the most rational. Reducing bitumen viscosity would make bitumen layer much easier to recess from sand grains, thus facilitating bitumen purification.

Among such methods, thermal and thermochemicals ones occupy a special place.

However, a previous study has shown that the energy consumption for $1{ }^{\circ} \mathrm{C}$ increase per a tonne of oil sand ore is 5 million Joules [8]. With it the energy intensive process unavoidably emits greenhouse gases which are an environmental concern.

Deposits located at a depth of less than $75 \mathrm{~m}$ can technically be surface mined, although most deposits are located at a depth of less than $50 \mathrm{~m}$ below grade. After mining, bitumen is separated from sand using a hot water extraction process that was patented in 1928 by Dr. Karl Clark.

Hupka et al. studied the importance of bitumen viscosity when processing U.S. oil sands by the addition of kerosene $[6,9,10]$. Their study concluded that the amount of kerosene added depends on processing temperature, original bitumen viscosity and oil sands grade. The authors showed that there is a good agreement between bitumen recovery and its viscosity. To achieve a bitumen recovery, greater than $90 \%$ of bitumen viscosity must be reduced to $0.5 \sim 2 \mathrm{~Pa} \cdot \mathrm{s}$ at the temperature of digestion, regardless of oil sands type, grade or 
origin, shown in Figure 2. In fact, if bitumen viscosity was to be controlled below $6 \mathrm{~Pa} \cdot \mathrm{s}$, bitumen recovery could keep by $80 \%$ or higher.

To achieve satisfactory separation in the water-based process, bitumen viscosity needs to be lowered to a critical value of $2 \mathrm{~Pa} \cdot \mathrm{s}$, regardless of the oil sands origin and characteristics [11].

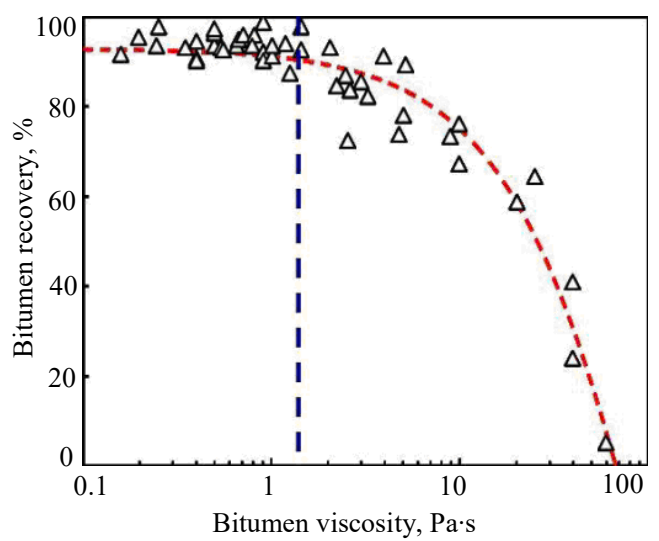

Fig. 2. Bitumen viscosity importance on bitumen recovery for water-based bitumen extraction process $[6,9,10]$.

With bitumen and water having similar densities, a difference in density is required in order to separate released bitumen from the slurry. The difference in density can be achieved by lowering the apparent bitumen density by aeration. Flotation has long been considered the single most important method in mining to separate minerals from ores hence. Dai and Chung have shown that the hydrophobicity of sand plays an important role in bitumen recession and liberation, with flotation efficiency increasing with decreasing hydrophobicity [12]. Hydrophobic solid particles tend to aerate more easily than hydrophilic particles hence; hydrophobic particles have an adverse effect on froth quality. Nguyen et al. confirmed the results of Chung showing that the interaction between an air bubble and a hydrophilic surface is repulsive, while the interaction between an air bubble and a hydrophobic surface is repulsive at a long distance and attractive at a short distance depending on the particle hydrophobicity [13]. Hupka et al. has shown that a more hydrophobic bitumen surface will readily attach to an air bubble and consequently improve flotation quality [6].

Miller and Misra have shown that the hot-water extraction process is influenced by many physical and chemical variables $[14,15]$. Variables such as bitumen viscosity, sand particle size distribution, temperature, $\mathrm{pH}$, time of digestion, flotation and degree of agitation, all influenced processability. The authors have concluded that the best flotation response is measured between $p H 7.8$ to 9.0 , relating to a maximum in the contact angle between an air bubble and bitumen surface.

Schramm et al. have shown that a reduction in viscosity by increasing the processing temperature or through the addition of diluents enhances bitumen separation for Athabasca oil sand ores [16].

Lelinski et al. have shown that for high bitumen viscosities, recession and liberation from the sand grain occur slowly, lowering the transfer rate between the sand and air bubble hence, lowering the efficiency of bitumen separation [17]. Overall, recoveries by temperature increase exceed those associated with solvent addition, which is in agreement with the study of Schramm et al. [17], who measured better recoveries from temperature increase compared to the addition of kerosene. 


\section{Technologies of natural bitumen extraction}

Surface mining describes an open-pit mine operation where the oil sands are excavated and transported by trucks to an extraction facility. At the extraction facility the oil sands are mixed with hot/warm water (temperature from 35 to $80^{\circ} \mathrm{C}$ [19]) containing sodium hydroxide (usually $\mathrm{NaOH}$ ). Traditionally, conditioning drums are used to release bitumen at $80^{\circ} \mathrm{C}$. At a temperature of $35-50^{\circ} \mathrm{C}$ and a $\mathrm{pH}$ slurry between $8.0-8.5$, bitumen recedes to form droplets that are separated from sand grains under fluid shear.

Fig. 3 shows a typical scheme of bitumen extraction for an open mine operation.

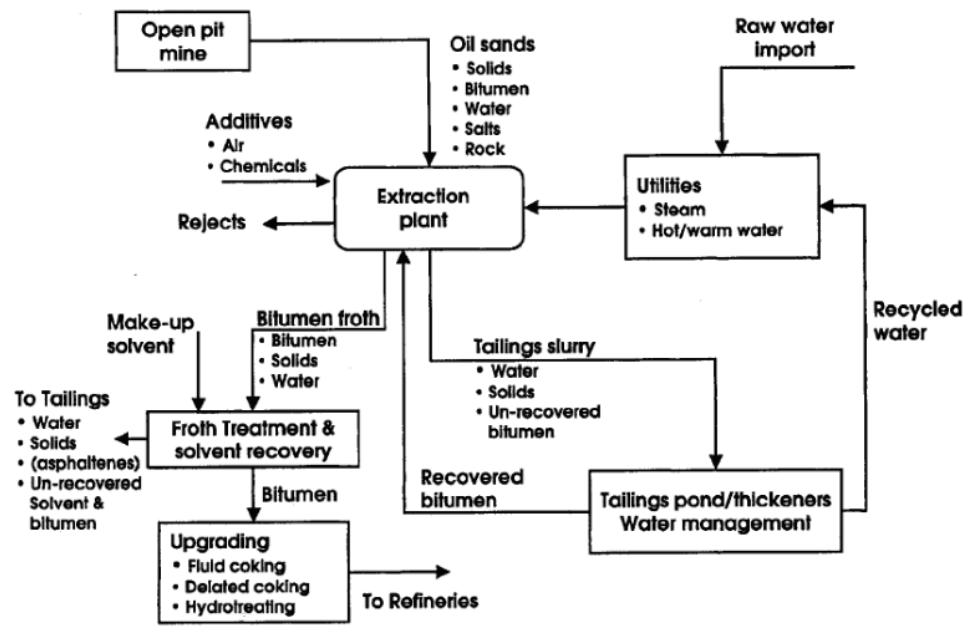

Fig. 3. Flow chart for the commercial water-based bitumen production process [3].

In order to enduce separation, air is drawn into the slurry to aerate the released bitumen. The combination of hot water and agitation releases bitumen from the oil sand, and causes tiny air bubbles to attach to the bitumen droplets. Aerated bitumen droplets have an apparent density less than water and hence float to form a 'rich' froth layer in bitumen. These droplets come to the reservoir surface. The recovered froth usually contains $60 \mathrm{wt} \%$ of bitumen, $30 \mathrm{wt} \%$ of water, and $10 \mathrm{wt} \%$ of solids. This process recovers about $90 \%$ of the bitumen. Coarse solids settle down to form tailings.

Further processing removes residual water and solids. Bitumen normally requires dilution with lighter hydrocarbons to make it transportable through pipelines. The bitumen is then transported and eventually upgraded into synthetic crude oil. (Bitumen upgrading to synthetic crude oil is comparable to conventional crude oil treatment using thermal and catalytic processing steps, such as coking, cracking or hydrotreating.) The synthetic crude oil is then piped to a refinery for further physical and chemical processing.

The quarry method is wide spread in Canada, where up to $20 \%$ of proven reserves of bitumen occur in sandy collectors at a depth of $50 \mathrm{~m}$. The remaining $80 \%$ of the bitumen is too deep to be mined. For deeper $(>75 \mathrm{~m})$ bitumen stocks, in-situ recovery methods are used. Most in-situ bitumen and heavy oil production comes from deposits occurring more than 400 meters below the earth surface.

The Steam-Assisted Gravity Drainage (SAGD) technology is believed one of the most effective and applicable [20]. To implement SAGD, two horizontal wells are drilled (one above the other at a distance of $3-7 \mathrm{~m}$ ). The steam is fed during 90 days into the wells and creates a heated-up zone, reducing the viscosity of bitumen (oil) and increasing mobility. The force of gravity directs condensed water and mobile hydrocarbons (bitumen) to the 
lower mining well. In the second phase, the extraction of bitumen (high viscosity oil) is carried out from the recovery well and steam is pumped into the inqection well. The heat exchanging process is constantly observed on the surface of division between the steam chamber and the cold stratum. As a result, the steam is being condensed and together with the heated oil flows down to the mining well under the gravity force action. SAGD has demonstrated that its operations can recover as much as $60 \%$ of the bitumen in-situ. SAGD wells operate at low steam pressure. But at the same time the roof of the productive formation should be a reliable barrier to prevent the loss of steam.

The commercial efficiency of the extraction method using solvents in a vapor state (VAPor EXtraction - VAPEX) has been also substantiated. This method is also implemented using two horizontal wells. Hot ethanes and propane-butane are pumped into the well located at the top of formation. Bitumen is being dissolved and under the gravity force action flows into the lower recovery well. This technology allows significantly reduce energy costs. It also allows to successfully develop collectors with a high clay content (the method using the steam in such conditions is unacceptable). The method allows to recover up to $60 \%$ of bitumen, but the rate of extraction is extremely low.

The new technology, N-Solv, is a modification of the VAPEX method. The technology uses propane heated to $50^{\circ} \mathrm{C}$. It requires at least $80 \%$ of energy, and propane can be reused. The solvent dissolves bitumen, and the heaviest components remain underground. The resulting product is obtained less viscous and can be transported immediately through the oil pipeline. The efficiency of the process has been greatly increased by removing of the methane admixture from propane. (Methane in the formation significantly degrades the heat exchanging process at the boundary of 'bitumen - solvent').

The processes co-injection of steam and solvent are also used: improved steam-gravity effect by the solvent - Expanding Solvent SAGD (ES SAGD) Process with addition of the solvent (SAP), alternating of injection of solvent and vapor- Steam Alternating. CHOPS technology [1] involves the 'cold' extraction of heavy oil along with sand. For this purpose, depression is created in the layer. So-called 'wormholes' are formed due to the destruction of weakly cemented rocks. Oil and sand come to the well through them. But the oil recovery ratio does not exceed $20 \%$ [1].

The 'cold' methods also include the VAPEX method. It involves the use of solvents [21 - 23]. A solvent (hydrocarbon solvents including ethane or propane) is pumped into the upper well instead steam. The bitumen is rarefied due to the solvent diffusion in it and flows to the recovery well under the action of gravitational forces. This method requires no water and is by $25 \%$ less in capital costs than the SAGD process. Operating costs are half than that of the SAGD process [20]. Two horizontal wells, placed one above the other, are used for this. The chamber-solvent is formed when the solvent is pumped into the upper well. The bitumen is rarefied and under the gravity action flows into the recovery well. This method does not require the use of water and is by $25 \%$ less in capital costs and by $50 \%$ less in operating costs compared with the process of SAGD [20]. The method, although it allows to achieve oil extraction up to $60 \%$, but the extraction rate is very low.

Currently, CSS (in CSS the steam is injected into the oil sands formation through a vertical well, recovering through the same well) and SAGD are the two methods used commercially in the Alberta oil sands industry.

From the technological point of view, steam is more efficient compared to hot water. Moreover, to reduce the thermal energy dissipation, it is more efficient to inject the steam into strata of considerable thickness. However, the steam injection creates a significant thermal load on the equipment. This causes additional risks of technical and technological complications. But the main problem of injection of water steam or hydrocarbon solvents is the heterogeneity of productive formations. This certainly leads to their breakthrough into the recovery wells and the need to apply diverter technologies (blocking gels, sediment- 
forming compositions).

Thus, the presently known mining technologies do not cover the entire depth range of natural bitumen deposits. Quarry mining can be carried out to a depth of $75 \mathrm{~m}$. The methods for extracting bitumen in site of its occurrence by injecting steam are applied starting at a depth of $200 \mathrm{~m}$ due to the danger of a breakthrough of the working agent on the surface. There are also problems of high energy consumption when using existing technologies and the negative impact on the environment. In this regard, the important task is to develop an effective method for this interval of bedding the bituminous sands.

\section{Technology of natural bitumen extraction through hydraulic mining by boreholes}

Well drilling is preceded by an in-situ extraction process. The hydrodynamic method is one of the ways to destroy mine rocks. It is carried out by a jet of high-speed liquid. By this method, significant thicknesses can be transferred to the bottom of wells. The mine rocks destruction by a jet of fluid containing abrasive particles in a suspended state is even more effective [24, 25]. However, the disadvantage of this method is the increased wear of equipment, the need for a large amount of abrasive material, a high probability of blocking the drill column when the drilling liquid circulation is suddenly stopped.

Physical and chemical technology of hydraulic borehole mining (HBM) minerals is well known in mining. In it, the hydraulic fluid energy is used to loosen the rock and convert it into a hydro-mixture (pulp) for extraction on the surface [26]. Penetrating into the massif due to filtration through pores and cracks, water reduces the strength of rocks, causing local hydrodynamic fractures and destruction [27].

The primary cutting mechanism in a HBM system is the conversion of kinetic energy of the water jet into impact pressure, acting on the rock. However, the rapid dissipation of the energy of the jet occurs in condition a flooded bottom [28]. Therefore, the distance from the nozzle to the ground is a determining parameter in the physical mechanism of energy transfer from the jet to the rock.

This requires the use of extended monitors and systems to control them. Also, the volume of recovery per well is limited due to the danger of roof decay. All this limits the sphere of application of hydraulic borehole mining (HBM) technological scheme.

In the works [29-32], a method for the development of marine gas hydrates deposits on the basis of hydraulic mining by boreholes is proposed. In the work [33], a variant of this method adaptation is proposed for the development of bituminous sands deposits.

The method (Fig. 4) includes the following operations:

1) uncovering of a bituminous sand seam by horizontal or inclined wells 8 heated by a working fluid - water with surface-active substances;

2) heating up of the well to reduce the bitumen viscosity by the circulation of the heattransfer medium through the central pipe and the ring space;

3) rock disintegration begining with the bottom of the well with a jet of liquid at a temperature above $100{ }^{\circ} \mathrm{C}$ (stream III) (With the help of hydraulic monitors 7 on the rods. In the working position, they occupy the position 8 perpendicular to the axis of the well. Rotating around the axis of the well, the hydraulic monitors gradually move to contact with the rock (the front of destruction). As a result, the rock becomes part of hydro-mixture);

4) gravity separation (behind the zone of turbulent mixing of hydro-mixture by the hydraulic monitoring jets) from the formed pulp into the sediment of the main mass of rock 2;

5) removal from the production of pulp (stream I) through the pulp picker 5, located in the arched part of the mine working to the separator 12; 
6) separation in the separator 12 of the solid residue from the pulp and its supply to the extraction unit.

The pressure of water for effective rock crushing is within the range of $5-20 \mathrm{MPa}$.

Given the phase equilibrium parameters of the system "water - steam", its temperature can be increased to $400-500 \mathrm{~K}$.

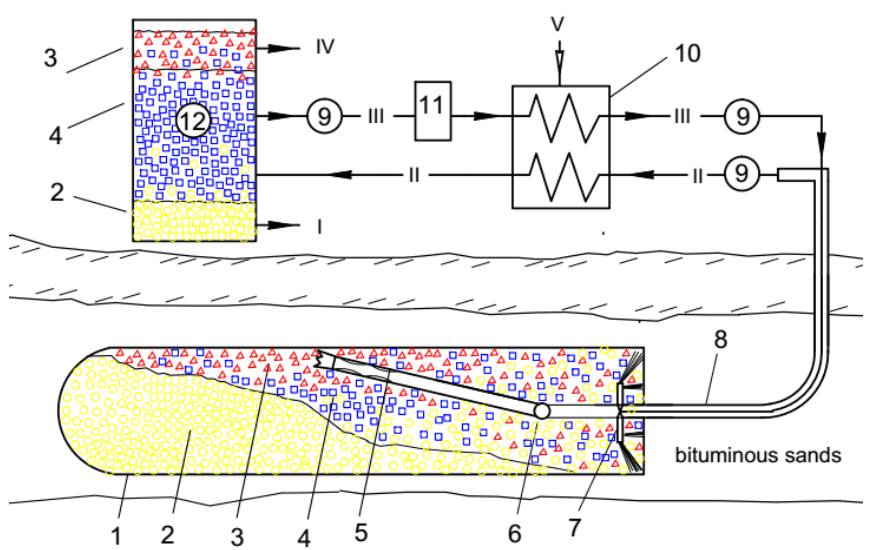

Fig. 4. Scheme of a method for the bitumen extraction from deposits of bituminous sands based on the HBM technology: 1 - development; 2 - sand sediment; 3 - pulp enriched in bitumen; 4 - water with clay fraction; 5 - a shank with a drilling bit and a pulp picker; 6 - hinge; 7 - device for hydraulic monitoring; 8 - well; 9 - pump; 10 - heating block; 11 - block of separation of solid phase; 12 separator; streams: I - sand sediment; II - a pulp enriched in bitumen; III - clay solution; IV bitumen; $\mathrm{V}$ - heat-transfer medium.

After the jet leaves the nozzle, part of the energy will be transferred to the added pulp mass [26].

It is also assumed that outside the hydraulic system, where the pressure corresponds to the formation (reservoir), the level of liquid overheating in the destruction zone will be sufficient for its boiling. The formation of water vapor bubbles in the rock pores will enhance the process of its disintegration.

After that, in the formed hydro-mixture, bitumen droplets will attach to the bubbles. Similar to processes carried out in extraction plants, the process of bitumen flotation to its arched part will occur in the mine working.

Thus, the method involves: rock conversion to the condition of the hydro-mixture, reducing the bitumen viscosity as a result of the thermal energy supply into mine working, the gravity separation of the hydro-mixture in the mine working and extraction of bitumen concentrate.

\section{Simulation of the rock heating process during well drilling}

At this stage of technology development, it is important to determine the thermodynamic parameters of the productive formation. Firstly, the thermodynamic parameters should be determined for the time interval from the beginning of the well drilling to the beginning of the rock disintegration (mining of bitumen). It is also necessary to solve the problem about stationary mode in the well during the well drilling phase.

So, further on, a mathematical model should be developed of the stationary mode (task 1) and dynamics (task 2) of the rock heating around the drill column. 
Formulation of the problem. Consider the process of drilling a horizontal well in the bituminous sand formation and its heating before the start of mining the bitumen. We have a drill column, which consists of two concentrically arranged pipes with radii $R_{1}=r_{\text {wat }}$ and $R_{2}=r_{\text {well }}$. The rock destruction device is located at its end. In the drilling process the drill column gradually penetrates into bituminous sand formation through a horizontal well. The heated water moves through the inner pipe to the rock destruction device. From it, a mixture of water and crushed rock (pulp) is returned through the ring (inter-tubular) space to the well head. The process is accompanied by heat exchange with the adjoining rock - it is heated. As a result, the viscosity of bitumen in this rock is reduced. This, in turn, reduces the coupling between its particles, and hence further simplifies its disintegration. We simulate the heating process of the rock, adjacent to the drill column (Fig. 5).

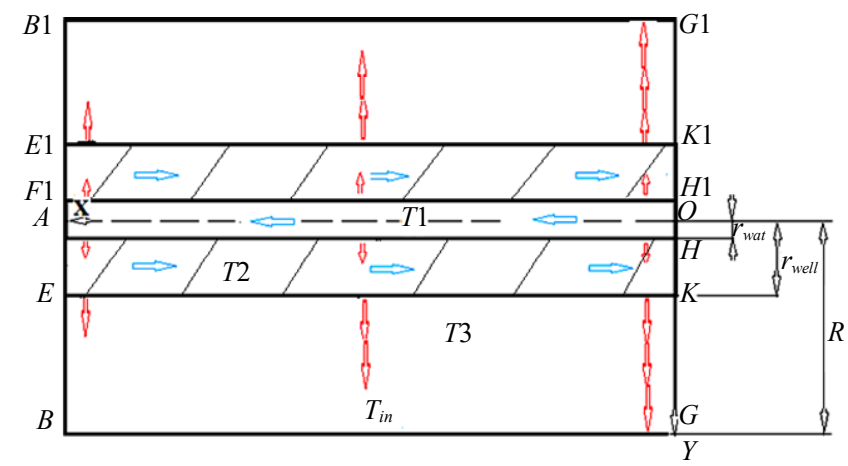

Fig. 5. Schematic representation of the heating process of rock during drilling of a horizontal well in a bituminous sand formation: $\longrightarrow-$ the direction of the fluid motion; $=-$ heat flow.

For this purpose, in a formation with an initial temperature $T_{i n}$, a specific volume should be selected of $B B_{1} G_{1} G$, in which the horizontal well is drilled with length $L$. The jet of warm water with the temperature $T_{\text {wat }}$ moves horizontally along the $O X$-axis through a pipe with radius $r_{\text {wat }}$. The device for the rock destruction, gradually disintegrating the rock in the volume $E E_{1} K_{1} K$, forms a horizontal well with radius $r_{\text {well }}$. The length of the well will be $x=O A=L$. In the drilling process, the part of mechanical energy is converted into thermal energy. Its value is neglected.

The drilling process will be accompanied by heat transfer. The heat in the central pipe and in the ring space is transferded by the fluid flow as a result of convective heat exchange. The inflow of warm water can be considered as a constant heat source $\left([w]=\mathrm{W} / \mathrm{m}^{3}\right)$ acting at the point $x_{1}$ (beginning of the pipe) during the drilling time $\tau$.

System of equations. The process of heat transfer in each of three volumes $(i=1,2,3)$ can be described by a differential equation in a cylindrical coordinate system:

$$
\frac{\partial T_{i}(x, r, t)}{\partial t}+v_{i x}(x, r, t) \frac{\partial T_{i}(x, r, t)}{\partial x}=-\frac{1}{\rho_{i} c_{i}} \operatorname{div} Q_{i}(x, r, t)+\frac{1}{\rho_{i} c_{i}} w_{i}(x, t),
$$

where $T_{i}, \rho_{i}, c_{i}$ are temperature, density and heating capacity of the corresponding medium; $v_{i}, Q_{i}$ and $w_{i}$ are velocity, heat flow density and density of external energy sources, respectively.

Boundaries of volumes are determined by the ratios:

- for the first volume: $0 \leq x \leq L, 0 \leq r \leq R_{1}$;

- for the second volume: $0 \leq x \leq L, R_{1} \leq r \leq R_{2}$;

- for the third, outer, volume: $0 \leq x \leq L, R_{2} \leq r \leq R$. 
Here, the value of $R$ determines the effective maximum radius of the outer volume of the rock to which the heat is spread.

In formula (1), it is taken into account that in this model the fluid velocity $v$ is directed along the well (along the $O X$-axis):

$$
v_{i x}(r, t)=v_{i x}(x, r, t) \equiv v_{i}(x, r, t), v_{i y}(r, t)=0, \quad v_{i z}(r, t)=0, \quad i=1,2,3,
$$

and the heat flow $Q$ is determined by the thermal conductivity $k$ of the corresponding medium:

$$
Q_{i}(x, r, t)=-k_{i} \operatorname{grad} T_{i}(x, r, t), i=1,2,3 .
$$

Differential equations (1) take into account the flow of water in the central pipe $(i=1)$ and the pulp in the ring space $(i=2)$. The velocities of the fluid in these areas $v_{1}(x, r, t)$ and $v_{2}(x, r, t)$ depend on the area of their cross section, the liquids viscosity, the pump productivity (or external pressure) and are determined by the Navier-Stokes equations for corresponding areas:

$$
\frac{\partial v_{i x}(x, r, t)}{\partial t}=-\frac{1}{\rho_{i}} \frac{\partial}{\partial x} P_{i}(x, r, t)-\frac{1}{\rho_{i}} \frac{1}{r} \frac{\partial}{\partial r}\left\{\eta_{i} r \frac{\partial v_{i x}(x, r, t)}{d r}\right\}, i=1,2,
$$

where $P_{i}(x, r, t)$ is pressure distribution, and $\eta_{i}$ is coefficient of the dynamic viscosity of the corresponding liquid. In equations (1) and (4), it is taken into account that the liquids are incompressible and move at low velocities. Also, in these equations, the dissipative coefficients of thermal conductivity and viscosity are generally functions of coordinates. This makes possible to use these equations for the case of large temperature gradients, that is, to take into account and investigate nonlinear effects.

Limit and initial conditions. In Problem 1, the stationary solution of the system of equations (1)-(4) is considered to explain the main features of this thermophysical problem. As the boundary conditions, the following ratios for temperature at the boundaries of volumes should be taken:

$$
T_{1}\left(r=r_{1}\right)=T_{2}\left(r=r_{1}\right) \text { and }\left.k_{1} \frac{\partial T_{1}}{\partial r}\right|_{r=r_{1}}=\left.k_{2} \frac{\partial T_{2}}{\partial r}\right|_{r=r_{1}} \ldots
$$

- at the boundary between the pipe with a smaller radius and a concentrically arranged pipe with larger radius:

$$
T_{2}\left(r=r_{2}\right)=T_{3}\left(r=r_{2}\right) \text { and }\left.k_{2} \frac{\partial T_{2}}{\partial r}\right|_{r=r_{2}}=\left.k_{3} \frac{\partial T_{3}}{\partial r}\right|_{r=r_{2}} \ldots
$$

- at the boundary between a pipe with a larger radius and an outside area:

$$
T_{3}\left(r=r_{3}\right)=T_{0} \text {. }
$$

- at the boundary of the outside area. Here $T_{0}$ is the ambient temperature.

For the velocity of viscous nonideal liquid, the immovability condition is executed at the boundaries:

$$
v_{1}\left(r=r_{1}\right)=0
$$

- on the inner wall of the pipe with a smaller radius

$$
v_{2}\left(r=r_{1}\right)=0
$$


- on the outer wall of the pipe with a smaller radius

$$
v_{2}\left(r=r_{2}\right)=0
$$

- on the inner wall of the pipe with a larger radius.

The boundary conditions for the temperature and velocity at the beginning of the pipes $(x=0)$ and the ends of the pipes $(x=L)$ will be considered in specific examples.

In Problem 2, the solution of the system of equations (1) - (4) will be considered, which gives a description of the thermal field evolution. As the initial conditions for temperature in all areas, the following should be taken:

$$
T_{1}(r, t=0)=T_{2}(r, t=0)=T_{3}(r, t=0)=T_{0} .
$$

The boundary conditions describe the ratios (5) - (10).

The presence of a closed system of equations (1) - (4) and the necessary boundary conditions (5) - (11) makes possible to solve the problem, that is to determine the temperature distribution in the allocated volume of bituminous sand (rock) during well drilling and under the stationary conditions.

Analytical studies of the physical model of the rock heating process during drilling of the horizontal well.

To solve the Problem 1 of stationary stable heat exchange in the simplest approximation, it should be performed the separation of variables and then, the radial coordinates of the equation and the results for the liquids velocities should be averaged.

As a result of this procedure, an equation for temperatures is obtained:

$$
\begin{gathered}
v_{1} \frac{\partial T_{1}(x, r)}{\partial x}=\chi_{1} \Delta T_{1}(x, r) ; \\
-v_{2} \frac{\partial T_{2}(x, r)}{\partial x}=\chi_{2} \Delta T_{2}(x, r) ; \\
0=\Delta T_{3}(x, r) .
\end{gathered}
$$

The coefficients of temperature conductivity are introduced into dependences (12) and (13):

$$
\chi_{1}=\frac{k_{1}}{\rho_{1} c_{1}} \text { and } \chi_{2}=\frac{k_{2}}{\rho_{2} c_{2}} .
$$

For averaged velocity values we have:

$$
\begin{aligned}
& v_{1}=\frac{1}{16} \frac{\Delta P}{\eta_{1}} \frac{r_{1}^{2}}{L_{1}} ; \\
& v_{2}=\frac{1}{16} \frac{\Delta P}{\eta_{1}} \frac{r_{1}^{2}}{L_{1}} .
\end{aligned}
$$

It is more suitable to start a solution from the last equation describing the outer area. As the simplest solution, the product of functions can be taken that are solutions of the corresponding Laplace equation:

$$
T_{3}(x, r)=T_{0}+\Delta T_{b}\left(1-\frac{x}{L}\right) \frac{\ln \left(\frac{r_{3}}{r}\right)}{\ln \left(\frac{r_{3}}{r_{2}}\right)}+D\left(x^{2}-\frac{r^{2}}{4}\right),
$$


where $\Delta T_{b}$ and $D$ are constants that will be determined from the boundary conditions.

In the second area, the temperature can be written as follows:

$$
T_{2}(x, r)=A\left(-\frac{x}{v_{2}}+\frac{r}{4 \chi_{2}}\right)+B \ln r+C \frac{-\chi_{2}}{v_{2}} \frac{x}{b^{2}}\left(1+\frac{r^{2}}{b^{2}}\right),
$$

where $A, B, C$ and $b^{2}$ are constants that will be determined from the boundary conditions.

In the first area, the temperature is written as follows:

$$
T_{1}(x, r)=T_{h}+E\left(\frac{x}{v_{1}}+\frac{r}{4 \chi_{1}}\right)+F \frac{\chi_{1}}{v_{1}} \frac{x}{a^{2}}\left(1+\frac{r^{2}}{a^{2}}\right),
$$

where $E, F$ and $a^{2}$ are constants that will be determined from the boundary conditions.

The last summands in (16) and (18) represent expansions by a small radius values of exact solutions of Poisson equations in a cylindrical coordinate system (Bessel function).

The computer model of the rock heating process by a horizontal well.

The numerical solution of Problem 1 about the stationary temperatures distribution for the established liquid and heat flows, as well as the solution of Problem 2 about the temporal temperature distribution, was initially executed on the basis of the complete system of equations (1) - (4), by standard methods. In this case, a stationary solution is obtained within a large time interval, when it can be assumed that the system has entered the stationary mode.

For a numerical solution of the system (1) - (4) with boundary conditions (5) - (11), a difference scheme was used for a cylindrical system of coordinates. In this case, the sametype equations in finite differences are specified for all areas. Their solution is made in an explicit scheme by the method of consistent calculation of time layers:

$$
\begin{gathered}
\frac{T_{i, j}^{(t+1)}-T_{i, j}^{(t)}}{\Delta t}+v \frac{T_{i+1, j}^{(t)}-T_{i-1, j}^{(t)}}{2 \Delta x}= \\
=\chi\left[\frac{T_{i+1, j}^{(t)}-2 T_{i, j}^{(t)}+T_{i-1, j}^{(t)}}{\Delta x^{2}}+\frac{T_{i, j+1}^{(t)}-2 T_{i, j+1}^{(t)}+T_{i, j-1}^{(t)}}{\Delta r^{2}}+\frac{T_{i, j+1}^{(t)}-T_{i, j-1}^{(t)}}{2 r_{j} \Delta r}\right],
\end{gathered}
$$

where $i$ is index numbering the nodes along the $O X$-axis; $j$ is index numbering the nodes along the radius $r ; \Delta t$ is grid spacing in time; $\Delta x$ and $\Delta r$ are corresponding coordinates $x$ and $r$.

The coordinates of nodes are specified by the ratio:

$$
\begin{gathered}
x_{i}=i \Delta x ; \\
r_{j}=\Delta r / 2+j \Delta r .
\end{gathered}
$$

This provides that the condition of temperature continuity is satisfied.

The condition for the flows continuity was taken into account in the boundary node as follows:

$$
T_{i, j+1}^{(t+1)}=T_{i, j}^{(t)}+\frac{k_{2}}{k_{2}}\left(T_{i, j}^{(t)}-T_{i, j-1}^{(t)}\right) .
$$

Examples of calculations of the thermal field evolution are represented in Figs. $6-8$. 


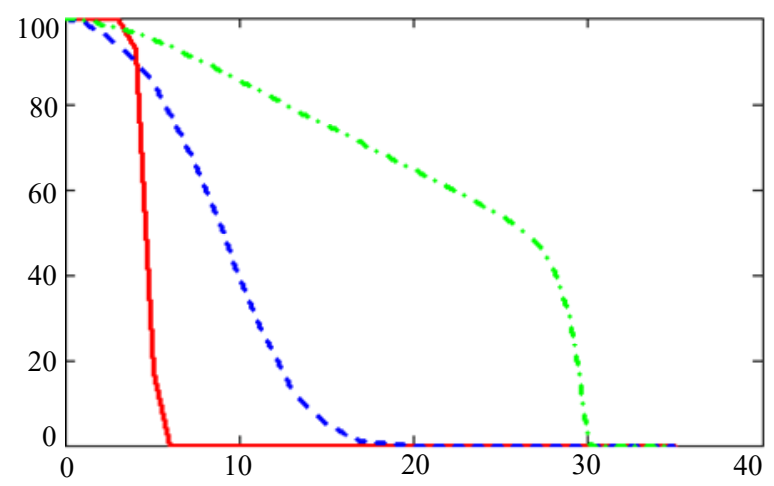

Fig. 6. Evolution of the temperature field on the axis of the drill column.

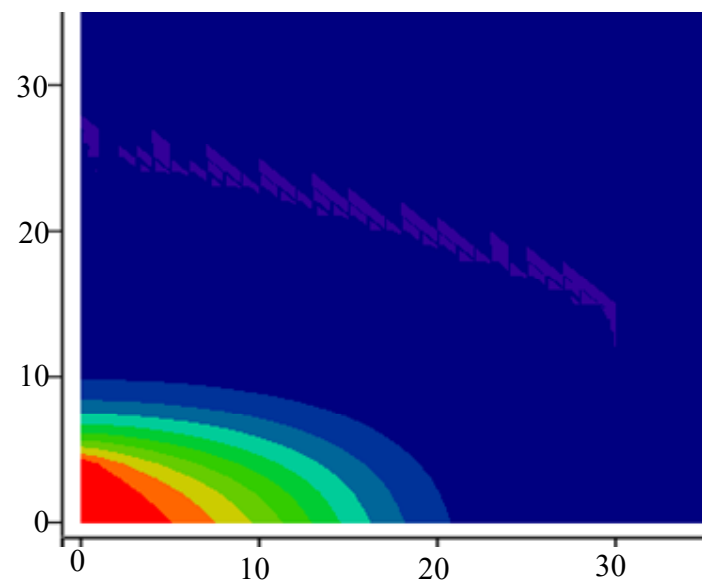

Fig. 7. Two-dimensional temperature distribution chart at the beginning of the process.

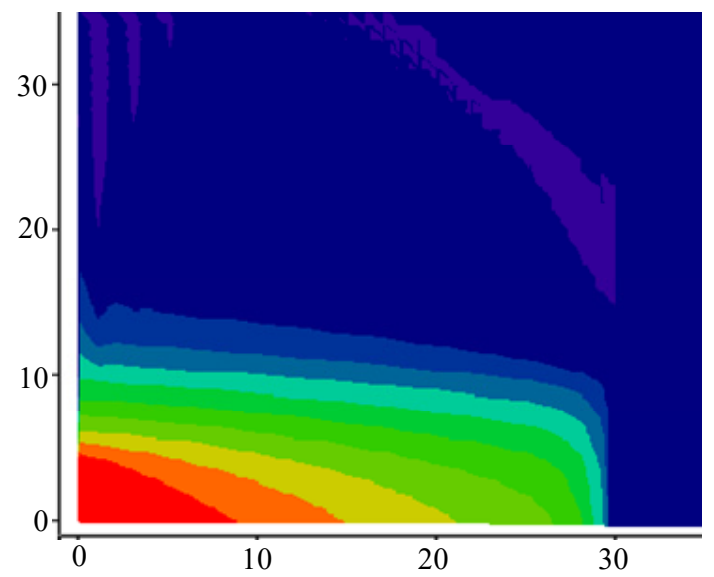

Fig. 8. Stationary temperature distribution.

Thus, the sequence of operations, provided in the method, allows preheating the rock adjacent to the well. As a result, the bitumen viscosity and the coupling of the rock particles is reduced. As a result, the efficiency of the bitumen separation process will improve and the cost of the rock destruction will decrease. 


\section{Conclusions}

The proposed method involves: rock conversion to the condition of the hydro-mixture, reducing the bitumen viscosity as a result of the thermal energy supply into mine working, the gravity separation of the hydro-mixture in the mine working and extraction of bitumen concentrate.

Analysis of the processes that will occur in the mine working, gives foundation to predict the rate of bitumen extraction according to this technology at the quarry method level.

Given the relatively low thermal rock conductivity, the possibility of thermal energy recuperation, low water consumption and a potentially high rate of bitumen recovery, this technology can be highly competitive.

Authors express their gratitude to Ivan Zezekalo, Professor, PhD, Director General of the Nadraspetstekhnolohii Firm for the help and consultation in performing the work.

\section{References}

1. Sadler, K.W. (1995). An EUB Review of In Situ Oil Sands Bitumen Production. SPE International Heavy Oil Symposium. https://doi.org/10.2118/30240-ms

2. Farouq, Ali S.M. (1974). Heavy Oil Recovery - Principles, Practicality, Potential, and Problems. SPE Rocky Mountain Regional Meeting. https://doi.org/10.2118/4935-ms

3. Masliyah, J.H., \& Gray, M.R. (2010). Oil Sands Extraction \& Upgrading-Intensive Short Course Notes. Calgary.

4. Takamura, K. (1982). Microscopic structure of athabasca oil sand. The Canadian Journal of Chemical Engineering, 60(4), 538-545. https://doi.org/10.1002/cjce.5450600416

5. Chilingar, G.V., \& Yen, T.F. (1978). Bitumens, Asphalts and Tar Sands. Development in Petroleum Science. https://doi.org/10.1016/s0376-7361(08)x7004-9

6. Hupka, J., Miller, J.D., \& Drelich, J. (2004). Water-Based Bitumen Recovery from Diluent-Conditioned Oil Sands. The Canadian Journal of Chemical Engineering, 82(5), 978-985. https://doi.org/10.1002/cjce.5450820513

7. Masliyah, J., Zhou, Z. J., Xu, Z., Czarnecki, J., \& Hamza, H. (2008). Understanding Water-Based Bitumen Extraction from Athabasca Oil Sands. The Canadian Journal of Chemical Engineering, 82(4), 628-654 https://doi.org/10.1002/cjce.5450820403

8. Cymerman, G.J., Ng, S., Siy, R., \& Spence, J. (2006). Energy Conservation Measures at Syncrude Oil Sand Processing Operations. Paper 2191. CIM Mining Conference \& Exhibition, Vancouver, BC, Canada.

9. Hupka, J., Miller, J.D., \& Cortez, A. (1983). Importance of Bitumen Viscosity in the Hot Water Processing of Domestic Tar Sands. Mining Engineering, 35(12), 1635-1641.

10. Hupka, J., \& Miller, J. D. (1993). Tar sand pretreatment with diluent. Mining, Metallurgy \& Exploration, 10(3), 139-144. https://doi.org/10.1007/bf03403016

11. Miller, K.A., Nelson, L.A., \& Almond, R.M. (2006). Should You Trust Your Heavy Oil Viscosity Measurement? Journal of Canadian Petroleum Technology, 45(04), 42-48. https://doi.org/10.2118/06-04-02

12. Dai, Q., \& Chung, K.H. (1996). Hot water extraction process mechanism using model oil sands. Fuel, 75(2), 220-226. https://doi.org/10.1016/0016-2361(95)00218-9

13. Nguyen, A.V., Evans, G.M., Nalaskowski, J., \& Miller, J.D. (2004). Hydrodynamic interaction between an air bubble and a particle: atomic force microscopy measurements. Experimental Thermal and Fluid Science, 28(5), 387-394. https://doi.org/10.1016/j.expthermflusci.2003.01.001

14. Miller, J.D., \& Misra, M. (1982). Concentration of Utah tar sands by an ambient temperature flotation process. International Journal of Mineral Processing, 9(3), 269-287. 
https://doi.org/10.1016/0301-7516(82)90033-3

15. Misra, M., \& Miller, J.D. (1991). Comparison of water-based physical separation processes for U.S. tar sands. Fuel Processing Technology, 27(1), 3-20. https://doi.org/10.1016/0378$\underline{3820(91) 90004-\mathrm{v}}$

16. Schramm, L.L., Stasiuk, E.N., \& Turner, D. (2003). The influence of interfacial tension in the recovery of bitumen by water-based conditioning and flotation of Athabasca oil sands. Fuel Processing Technology, 80(2), 101-118. https://doi.org/10.1016/s0378-3820(02)00224-2

17. Lelinski, D., Drelich, J., Miller, J.D., \& Hupka, J. (2008). Rate of Bitumen Film Transfer from a Quartz Surface to an Air Bubble as Observed by Optical Microscopy. The Canadian Journal of Chemical Engineering, 82(4), 794-800. https://doi.org/10.1002/cjce.5450820418

18. Schramm, L.L., Stasiuk, E.N., Yarranton, H., Maini, B.B., \& Shelfantook, B. (2003). Temperature Effects From the Conditioning and Flotation of Bitumen From Oil Sands in Terms of Oil Recovery and Physical Properties. Journal of Canadian Petroleum Technology, 42(08), 5561. https://doi.org/10.2118/03-08-05

19. Liu, J., Xu, Z., \& Masliyah, J. (2008). Interaction between Bitumen and Fines in Oil Sands Extraction System: Implication to Bitumen Recovery. The Canadian Journal of Chemical Engineering, 82(4), 655-666. https://doi.org/10.1002/cjce.5450820404

20. Canada's Oil Sands, Opportunities and Challenges to 2015. An Energy Market Assessment. Canada: National Energy Board.

21. Azin, R., Kharrat, R., Ghotbi, C., \& Vossoughi, S. (2005). Applicability of the VAPEX Process to Iranian Heavy Oil Reservoirs. In Proceedings of SPE Middle East Oil and Gas Show and Conference. https://doi.org/10.2523/92720-ms

22. Das, S.K. (1998). Vapex: An Efficient Process for the Recovery of Heavy Oil and Bitumen. SPE Journal, 3(03), 232-237. https://doi.org/10.2118/50941-pa

23. Yazdani, Ali J., \& Maini, B. (2004). Effect of Drainage Height and Grain Size on the Convective Dispersion in the Vapex Process: Experimental Study. Proceedings of SPE/DOE Symposium on Improved Oil Recovery. https://doi.org/10.2523/89409-ms

24. Sulakshin, S.S., \& Chubik, P.S. (2011). Razrushenie gornykh porod pri provedenii geologorazvedochnykh rabot. Tomsk: Tomskiy politekhnicheskiy universitet.

25. Maksimov, V.I. (1971) Novye sposoby bureniya skvazhin. Moskva: VIEMS.

26. Arens, V., Babichev, A., Bashkatov, A., Gridin, O., Khrulev, A., \& Khcheyan, G. (2007). Borehole Hydro-Mining. Proc. Manual: Mining Book.

27. Rehbinder, G. (1980). A Theory about Cutting Rock with Water Jet. Rock Mechanics, 12(3-4), 247-257. http://dx.doi.org/10.1007/bf01251028

28. Helgerud M. B. (2001). Wavespeeds in gas hydrate and sediments containing gashydrate: A laboratory and modeling study. Ph.D. Thesis. Stanford, USA: Stanford Univ. Press.

29. Pedchenko M., Pedchenko L. (2017). Analysis of gas hydrate deposits development by applying elements of hydraulic borehole mining technology. Mining of Mineral Deposits, 11(2), 52-58. https://doi.org/10.15407/mining11.02.052

30. Pedchenko, L., \& Pedchenko, M. (2012). Substantiation of Method of Formation of Ice Hydrate Blocks with the Purpose of Transporting and Storage of Hydrate Gas. Naukovyi Visnyk Natsionalnoho Hirnychoho Universytetu, (1), 28-34.

31. Bondarenko, V., Svietkina, O., \& Sai, K. (2017). Study of the formation mechanism of gas hydrates of methane in the presence of surface-active substances. Eastern-European Journal of Enterprise Technologies, 5(6 (89)), 48-55. https://doi.org/10.15587/1729-4061.2017.112313

32. Bondarenko, V., Maksymova, E., \& Koval, O. (2013). Genetic classification of gas hydrates deposits types by geologic-structural criteria. Annual Scientific-Technical Colletion - Mining of Mineral Deposits, 115-119. https://doi.org/10.1201/b16354-21

33. Pedchenko, M., \& Pedchenko, L. (2018) Prospects the application of hydraulic borehole mining technology for the development of unconventional hydrocarbon deposits. E3S Web of Conference. (60), 00018. https://doi.org/10.1051/e3sconf/20186000018 